\title{
DIRECT MEASUREMENT OF THE SIZE OF THE LARGE KUIPER BELT OBJECT (50000) QUAOAR
}

\author{
Michael E. Brown and Chadwick A. Trujillo \\ Division of Geological and Planetary Sciences, California Institute of Technology, Pasadena, CA 91125; mbrown@caltech.edu \\ Received 2002 October 7; accepted 2003 December 23
}

\begin{abstract}
We imaged the recently discovered bright Kuiper belt object (50000) Quaoar with the Hubble Space Telescope High Resolution Camera to directly determine its size. The point-spread function (PSF) of each of 16 images was carefully measured from a field star 13" from Quaoar, and the expected PSF at the location of Quaoar was convolved with Quaoar's motion vector and a model resolved disk. A least-squares analysis was performed to find the best-fit disk size. The apparent diameter of Quaoar was resolved as $40.4 \pm 1.8$ milliarcseconds. Accounting for the uncertainty due to an unknown limb-darkening function, the size of Quaoar is $1260 \pm 190 \mathrm{~km}$ with red and blue albedos of $0.092_{-0.023}^{+0.036}$ and $0.101_{-0.024}^{+0.039}$, respectively. These albedos are significantly higher than the canonically assumed value of $4 \%$. Quaoar is the largest currently known minor planet.
\end{abstract}

Key words: minor planets, asteroids — techniques: image processing

\section{INTRODUCTION}

Many hundreds of Kuiper belt objects (KBOs) are currently known, and an understanding of their history, dynamics, and surface properties is gradually emerging. The sizes of KBOs are, however, almost entirely unknown. To date, only two of the largest objects have had their sizes reliably measured. These measurements have used the technique of radiometry, wherein a temperature for the object is assumed from the heliocentric distance and a measurement of thermal emission is then converted to an emitting area and, therefore, object size. Emission has been detected at a wavelength of $0.85 \mathrm{~mm}$ from (20000) Varuna (Jewitt, Aussel, \& Evans 2001) and at $1.3 \mathrm{~mm}$ from $2002 \mathrm{AW}_{197}$ (Margot et al. 2002) at fluxes of $2.81 \pm 0.85$ and $1.05 \pm 0.30 \mathrm{mJy}$, respectively, implying sizes of $900_{-145}^{+124}$ and $890_{-130}^{+115} \mathrm{~km}$. The $R$-band albedos implied from these measurements, combined with the optical fluxes of the objects, are $0.07_{-0.02}^{+0.03}$ and $0.10_{-0.02}^{+0.04}$, respectively, higher than the canonical $4 \%$ value assumed for KBOs in most studies. Some of the largest errors in these analyses are the systematic errorsunaccounted for in the above error bars-associated with understanding the thermal emission of the object.

The discovery of the extremely bright KBO (50000) Quaoar presented the opportunity to attempt the measurement of the size of a KBO in a direct and entirely different manner. At a magnitude of $R=18.5$, a geocentric distance of $43.4 \mathrm{AU}$, and an assumed albedo of 4\%, Quaoar's diameter would be $1910 \mathrm{~km}$, almost as large as that of Pluto. Even assuming a higher albedo of $10 \%$, Quaoar would be $1210 \mathrm{~km}$, about half the size of Pluto and larger than its moon Charon. Objects of these sizes at the distance of Quaoar should be directly resolvable with the Hubble Space Telescope. At a wavelength of $435 \mathrm{~nm}$, the High Resolution Camera (HRC) has a resolution of 40 milliarcseconds (mas), corresponding to $1250 \mathrm{~km}$ at the distance of Quaoar. With careful measurement of the point-spread function (PSF), objects with diameters even smaller than this resolution are resolvable. We thus undertook HRC observations of Quaoar to determine the size of this potentially large $\mathrm{KBO}$ and to provide an alternative method for the size measurement of the largest KBOs.

\section{OBSERVATIONS}

We obtained $16120 \mathrm{~s}$ exposures of Quaoar using the High Resolution Camera (HRC) of the Advanced Camera for Surveys in the F435W filter on 2002 July 29 in one orbit of the Hubble Space Telescope (HST). The observations began at UT 0450 on 2002 July 29 , and one observation was obtained every $171 \mathrm{~s}$. The timing of the observations was chosen so that Quaoar would be a few arcseconds from a moderately bright field star, and the telescope was tracked at the sidereal rate so that accurate PSF measurements could be obtained. The motion of Quaoar caused a smearing of about 60 mas of the image of Quaoar during each $120 \mathrm{~s}$ observation, a factor that needed to be taken carefully into account in the analysis of its size.

The star had a brightness of $32,650 \pm 170$ counts $(B=$ $18.47 \pm 0.01)$ in each of the images and was single, well exposed, and not saturated. Quaoar had a brightness of $9920 \pm 50$ counts $(B=19.76 \pm 0.01)$ and traveled across a field uncontaminated by detectable background stars or galaxies in the stacked images. These fluxes were determined from our full PSF-fitting analysis, described below.

\section{PSF DETERMINATION}

\subsection{Field Star PSF}

Determination of the angular size of Quaoar is dependent on an accurate measurement of the PSF of the field star. This measurement is complicated by two factors. First, at a wavelength of $435 \mathrm{~nm}$ the HRC PSF is undersampled. With a FWHM of 40 mas and a pixel size of $\sim 25$ mas, the core of the PSF changes noticeably depending on the precise subpixel position of a source, so direct measurement of the stellar PSF and translation to Quaoar at different subpixel positions would be imprecise. Second, the HRC images onto a distorted field on which the distortion changes with field position. Thus, even if a precise PSF could be measured at the position of the field star, the PSF would differ at the location of Quaoar. The change of the PSF over the $13^{\prime \prime}$ distance from the field star to Quaoar is likely small, but nonetheless, a reliable measurement of the angular size of Quaoar needs to take even this small variation into account. 
To accommodate the difficulties of PSF measurement with the HRC, we used the PSF simulation software Tiny Tim ${ }^{1}$ to help measure the PSF of the field star for each image (Krist \& Hook 2001). Tiny Tim simulates PSFs of HST images through direct computation of the diffraction pattern caused by passage through the entire optical system. The HRC is a new instrument on HST and the then current version of Tiny Tim (6.0) used only a preflight ray trace of the instrument; it thus needs to be extensively validated before modeled PSFs are used. Examination of the field star images demonstrated that the PSF of HST during these observations was time-variable. HST suffers from a well-known temporal focus shift, known as "breathing," induced by thermal expansion and contraction of the secondary support structure. Accurate characterization of these shifts and any other shifting aberrations was also critical to precise PSF modeling.

Initial tests with Tiny Tim showed that focus shifts over the one orbit of observation are the single largest contributors to PSF change. The total focus change corresponds to about $7 \mu \mathrm{m}$ of secondary shift. In addition, it was clear from the residuals of data minus model that significant and relatively constant trefoil (known as "clover" in Tiny Tim) was present in the image PSFs and that varying degrees of astigmatism and coma also occurred in the images. We therefore concluded that the proper procedure was to perform a least-squares fit for the first eight relevant Zernike terms in each image (defocus, two astigmatisms, two comae, two trefoils, and spherical aberration) using the field star.

In addition to these terms, the PSF of HST is affected by pointing jitter. Examination of the jitter logs for these observations shows that the jitter is generally well behaved, with rms values between 2.5 and 6.5 mas (with one outlier at 9.0 mas). As long as the jitter is smoothly distributed, as in these observations, it will be indistinguishable from the low-order Zernicke terms in the PSF and can be ignored as a separate factor in our PSF. In doing so, our absolute values for defocus error or astigmatism, for example, may be slightly off, but the resulting PSF model, which is what is used for the analysis, will still be correct.

The full procedure to create a best-fit Tiny Tim model of the PSF on one image proceeded as follows: First, Tiny Tim was used to generate a 5-times supersampled, geometrically distorted PSF at the location of the field star using nominal parameters for the first eight Zernike terms. This PSF was resampled with varying subpixel centering and convolved with the appropriate CCD charge diffusion kernel. A least-squares minimization between the model and the data was then performed to find the best-fit subpixel centering and total flux value for the nominal PSF. The Tiny Tim parameters were then modified by hand to create new PSFs with the Zernike terms varied one by one, from defocus to spherical aberration, solving for a best-fit subpixel center and total flux for each new PSF. At each step a least-squares optimal magnitude of the Zernike term was found, until all eight terms were determined. The process is CPU intensive and involves the testing of many varying PSF models, but the process is simplified by the fact that the Zernike polynomials are orthonormal, so that fitting of each term is independent of the others (a fact that we empirically verified after the fact by testing small variations in all parameters). Figure 1 shows the Zernike terms of the best-fit model determined from the field star for all 16 images. The defocus

\footnotetext{
1 Tiny Tim is available at http://www.stsci.edu/software/tinytim.
}

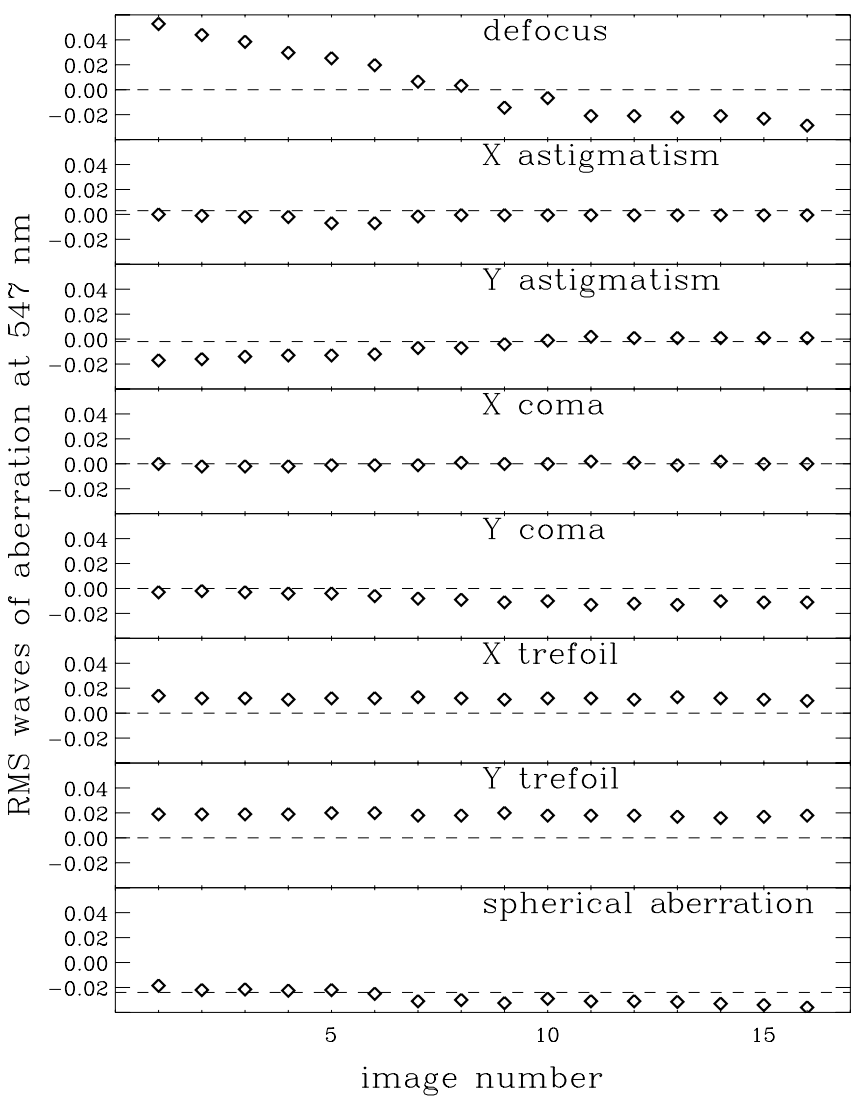

FIG. 1.-Magnitude of the Zernike terms determined from the field star in each image, in units of rms waves of aberration at $547 \mathrm{~nm}$. The dashed line shows the nominal Tiny Tim expected value for each term. For defocus, 0.011 waves of aberration correspond to $1 \mu \mathrm{m}$ of secondary focus shift. The smooth change in defocus over the orbit of observation is consistent with the wellknown temperature-induced secondary position changes ("breathing") of HST. Coma, astigmatism, and spherical aberration shift in concert with focus. Trefoil, which causes the largest PSF mismatch of any term other than defocus, is large and constant throughout the observations.

smoothly varies from $5 \mu \mathrm{m}$ out of focus to $2 \mu \mathrm{m}$ past optimal focus, a typical behavior owing to breathing of the telescope secondary. In addition, the astigmatism, coma, and spherical aberration vary in concert with the focus. Of all terms, only trefoil appears to be constant. This large, relatively static trefoil term causes the largest deviation from the data PSF of any term other than defocus.

The final match between the data and the PSF model is excellent. Figure 2 shows one of the 16 stellar images along with the final Tiny Tim model and the residuals. In this case, the residuals are indistinguishable from Poisson plus readout noise. In other cases, higher order aberrations occur at extremely low levels, but as we will later demonstrate, these residuals are sufficiently small as to not affect our measurement of the angular size of Quaoar.

\subsection{Modeled PSF Verification}

No model of a real PSF will perfectly represent the true PSF, and thus a test to determine whether imperfections in our modeled PSF can in any way mimic a resolved object is crucial. Our original choice of eight Zernike terms to model was based in part on the hope that by including all terms up to the first two spherically symmetric terms (defocus and spherical aberration) any remaining aberrations in the PSF would not mimic a resolved object. We tested that hypothesis by attempting to 


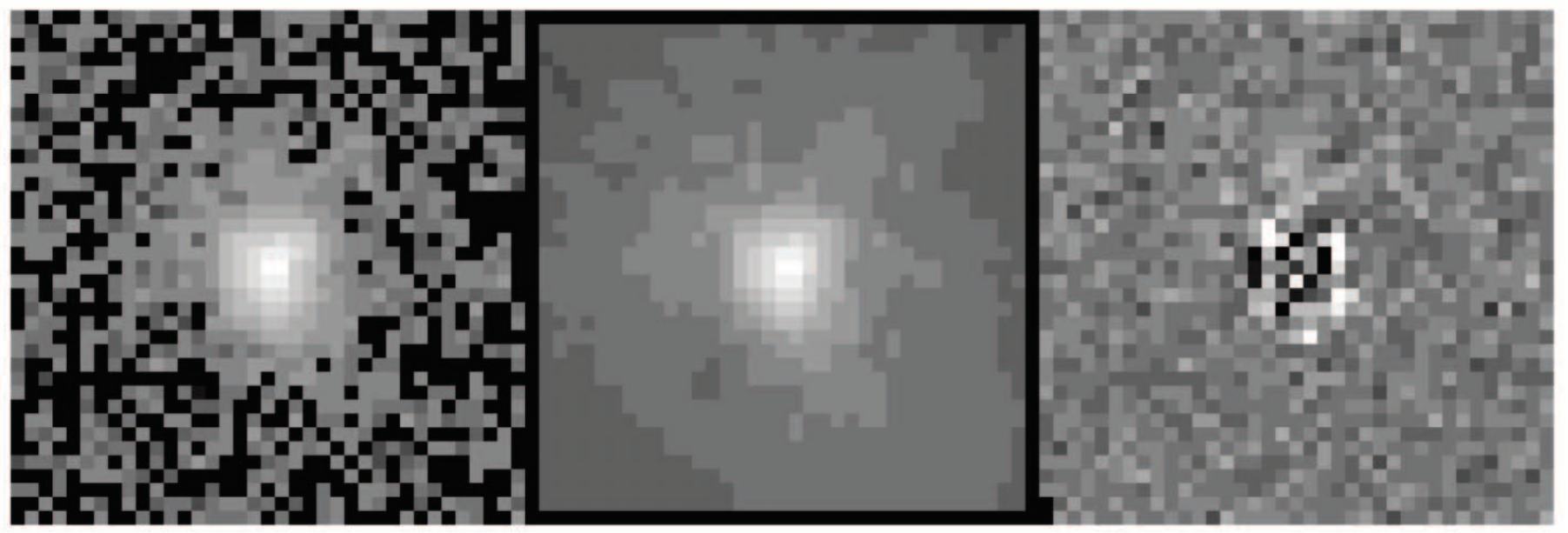

FIG. 2.-Comparison of one of 16 images of the field star (left), the best-fit Tiny Tim PSF (middle), and the residuals (right). The data and model share the same logarithmic scale. The residuals are linearly scaled from $\pm 1 \%$ of the maximum of the model image. The residuals are consistent with read plus Poisson noise.

measure the size of the star (which should be a point source) in each of the 16 images using the method that we will use to measure the size of Quaoar. We describe this method in detail below, but in Figure 3 we show the measured angular size of the star in each image to demonstrate that in 10 out of 16 cases the star is indistinguishable from a point source convolved with the derived model PSF (as detailed below, our most finely sampled PSF model has a pixel scale of 12.1 mas, so all objects from size 0 to 12 mas appear identical to us). In the remaining cases, the measured size of the PSF is either 13 or 14 mas, which are our first two measurable sizes above a point source. No correlation is seen between the magnitude of the jitter and the derived size of the star, supporting our contention that jitter is indistinguishable from other PSF effects. We believe that these small mismatches between the model and the data are due to limitations caused by a finite signal-to-noise ratio. We thus conclude that in at least 10 cases, our PSF models are sufficiently well matched to the data that they do not introduce any more than 12 mas artificial apparent size to point sources and

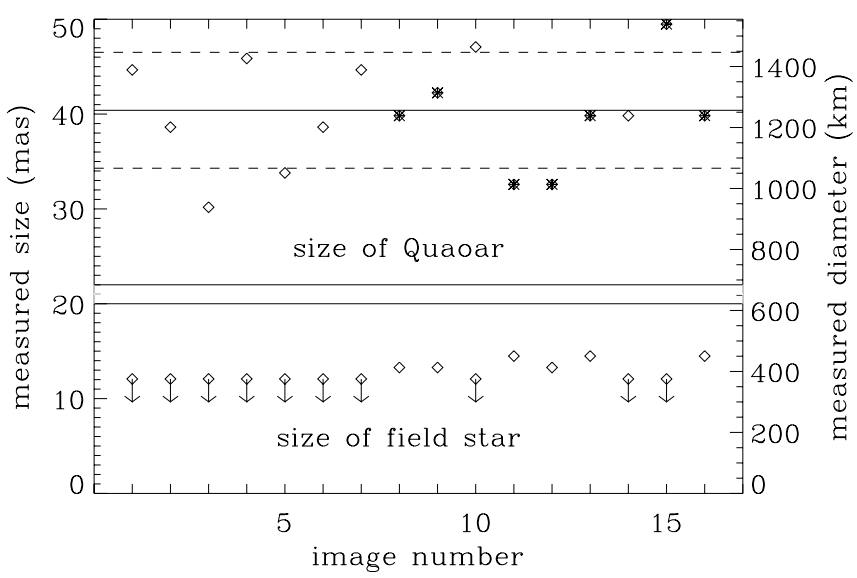

FIG. 3.-Angular size and linear size measured for the field star and for Quaoar in the 16 images. The six images for which we measured a finite size for the star (Nos. 8, 9, 11, 12, 13, and 16) and the one image for which we find a cosmic-ray hit near the center of Quaoar (No. 15) are marked and not used for further analysis, though their inclusion does not affect the final results. For the remaining 10 images, the average size of Quaoar is $40.4 \pm 1.8$ mas, or $1260 \pm 55 \mathrm{~km}$. Taking into account systematic effects the true errors are larger, leading to a result of $1260 \pm 190 \mathrm{~km}$, which is shown by the range of the dashed lines. that any size we measure greater than 12 mas in these 10 images for Quaoar is indeed intrinsic to the source. The six images with slightly worse PSF fits were discarded from further analysis, though it is shown below that their inclusion does not affect the final result.

\subsection{Field-dependent Distortion Verification}

Accurate knowledge of the PSF at the position of Quaoar requires that we trust that if Tiny Tim produces a PSF that matches the field star, it will also produce a PSF that matches Quaoar $13^{\prime \prime}$ away. To test if this ability is indeed the case, we examined HRC F435W images of 47 Tuc obtained in 2002 April for flat-field stability calibration purposes. We used our method described above to model the PSF of a star at the same position of our field star and to model the PSF of a star at the position of Quaoar. The parameters obtained for the Zernike terms are indistinguishable, suggesting that Tiny Tim's preflight incorporation of field distortion provides an excellent model for HRC distortions over these small distances. As a further check, we used the PSF model produced for the location of the field star, created a PSF at the location of Quaoar with the same parameters, and attempted to measure the angular size of a star at the location of Quaoar. We again find that the star at the location of Quaoar is indistinguishable from a point source. These tests lend confidence to our ability to determine the PSF at the location of our field star and trust the subsequent PSF model at the location of Quaoar.

\section{THE SIZE OF QUAOAR}

For each image we now have an accurate model of the PSF at the position of Quaoar. Theoretically we could deconvolve the data with this PSF to determine the size and motion of Quaoar, but without significantly more sampling of the data such a deconvolution will not provide reliable results. We chose instead to determine the size of Quaoar by forwardmodeling the effects of observing a resolved disk with the PSF determined at the position of Quaoar.

The first step was to determine the motion of Quaoar in each image. Quaoar moves along a curved path resulting from a combination of orbital motion, terrestrial parallax, and $H S T$ parallax. The $x$ and $y$ centroid positions as functions of time were fitted to third-order polynomials to smoothly define the motion of Quaoar. The amount of motion during each 
observation was determined by the derived difference in position at the beginning and end of each observation. The mean motion in the distorted image plane in the $x$-direction is 0.90 pixels during one observation, while the mean motion in $y$ is 2.2 pixels.

We then modeled each image as a geometrically distorted convolution of the model PSF and a model disk convolved with the motion vector. We used as the nondistorted model PSF the intermediate Tiny Tim output of the model PSF at a pixel scale of 12.1 mas pixel $^{-1}$.

To forward-model an image of an object of diameter $d$ mas, we first created an image of a 100 pixel diameter Lambert sphere (different potential limb-darkening functions for the disk are discussed below) and then resampled this image to a size of $d / 12.1$ pixels, creating a supersampled image of a Lambert sphere at a pixel scale of 12.1 mas. This model image was then convolved with the intermediate-output Tiny Tim model PSF at a pixel scale of 12.1 mas, as derived from the stellar image above, and this model disk image was appropriately geometrically distorted by Tiny Tim. Finally this model distorted image was convolved with the measured motion vector to create a supersampled, geometrically distorted, motion-smeared model image of a disk of diameter $d$. As was done above for the PSF stars, this supersampled model image was then resampled to the image pixel scale with various subpixel center positions, where it was convolved with the CCD charge transfer kernel. A leastsquares minimization between the final model image and the data was performed to find the best subpixel $x$ and $y$ center of the image and the total flux of the image. The entire procedure was repeated while varying the diameter $d$ of the model disk until the minimum least-squares best value of $d$ was obtained for each of the 16 images.

The model image fits to the data are generally excellent, with the exception of one image that suffered a cosmic-ray hit near the core of the PSF and was therefore not included in further analysis. Figure 4 shows a typical image of Quaoar compared with the modeled image and residuals.

The best-fit values for $d$ are shown in Figure 3. The mean of the nine reliable measurements is 3.34 supersampled 12.1 mas pixels with a standard deviation of 0.43 mas for a best-fit angular size of $40.4 \pm 1.8$ mas. At a distance of $42.9 \mathrm{AU}$, this size corresponds to a diameter of $1260 \pm 55 \mathrm{~km}$. If the five rejected images for which a finite size for the PSF star was measured are included, the final size is only $30 \mathrm{~km}$ smaller, suggesting that the small PSF mismatches that led us to reject these images actually have little effect on the final answer. From the analysis above, we placed an upper limit of 12 mas on the systematic errors inherent in the PSF model. If this potential PSF size is added in quadrature to the measured size, we find a potential systematic error of about 5\%. Below we find that other systematic effects dominate the final measurement uncertainty.

The small scatter in these nine independent measurements is a testament to the accuracy of the PSF models created from extensive Tiny Tim modeling of the field star. Confidence that this level of accuracy is warranted comes from the work of Noll et al. (1995), who performed similar measurements of the size of the near-Earth asteroid (4179) Toutatis. The angular size of Toutatis was similar to the size measured for Quaoar, and the HST measurements were consistent with independent radiometric measurements.

\section{SYSTEMATIC SOURCES OF ERROR}

The measurement of the size of Quaoar is remarkably precise; the random error bars of $4 \%$ are significantly smaller than those of $15 \%$ obtained for (2000) Varuna (Jewitt et al. 2001) and $13 \%$ obtained for $2002 \mathrm{AW}_{197}$ (Margot et al. 2002) using thermal radiometry. The main sources of error in the size of Quaoar, however, are likely to be systematic rather than random.

One potentially large source of systematic error is the unknown limb-darkening function of Quaoar. For our initial measurement, we assumed that the disk of Quaoar reflected as a Lambert surface and therefore had a limb-darkening function of $I=\cos ^{2} \alpha$, where $I$ is the brightness of a surface element at angle $\alpha$ from the subsolar point (assumed to be the same as the sub-observer point). For a Lambert sphere with diameter $d$, half the total light falls within a diameter of $0.6 \mathrm{~d}$. Experimentation with other limb-darkening functions suggests that these functions will change the derived size approximately proportionally to the change in half-total light diameter. For example, if Quaoar has a limb-darkening function closer to lunar, the halftotal light diameter would be approximately $0.7 d$ and the true size of Quaoar would be $1090 \mathrm{~km}$. If Quaoar instead has a surface with a strong specular reflection, it could have a much smaller half-total light diameter and actually be larger. The true limb-darkening function of such an icy outer solar system body is unknown. We take as representative errors the range from the Lambert to the lunar limb-darkening function and estimate an error for this unknown of $15 \%$.

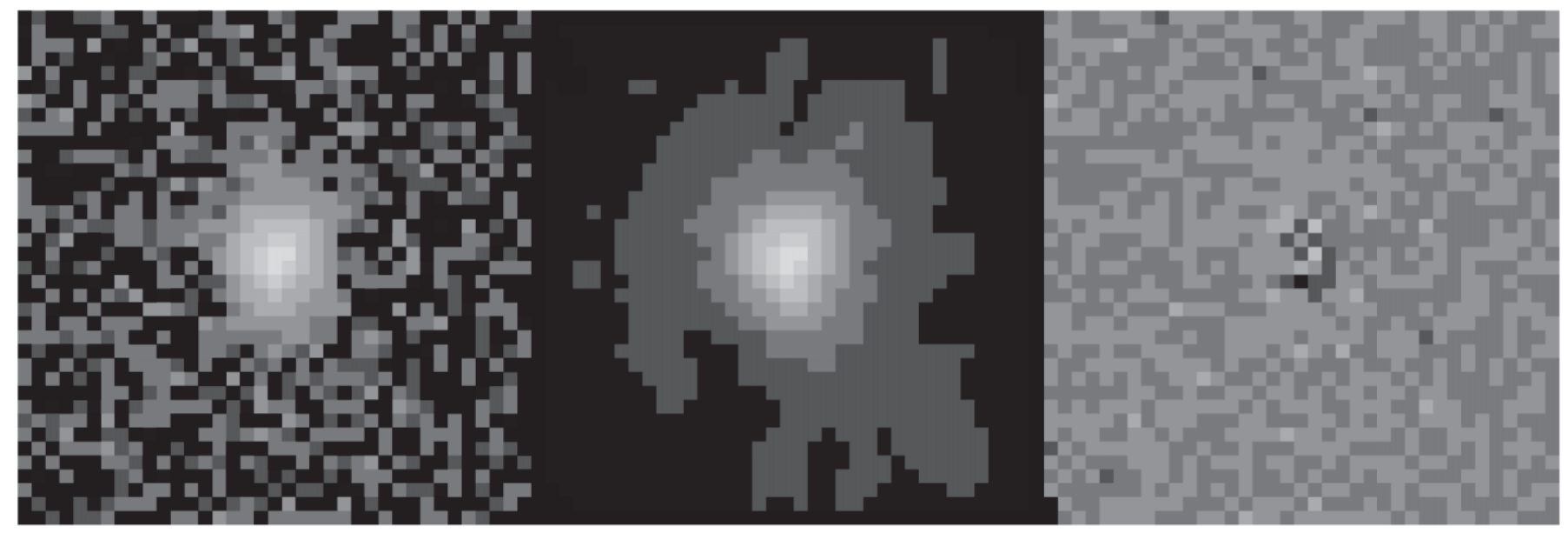

Fig. 4. - Comparison of one of 16 images of Quaoar (left), the best-fit model of a moving Quaoar of size 39 mas (middle), and the residuals (right). The data and models share identical logarithmic scales. The residuals are linearly scaled from $\pm 1 \%$ of the maximum of the model image. 
Another potential source of error could be a nonspherical shape or nonuniform surface. In our analysis we have assumed that Quaoar is a round and featureless body. Light-curve measurements show, however, that Quaoar has a 20\% intensity variation over a period of many hours (Trujillo \& Brown 2004). Such a variation could be due either to surface features or to a nonspherical shape, though a body of this size with a moderate rotation rate would be expected to be spherical. We nonetheless consider both effects.

First we model an ellipsoid with axes in the ratio 1.2:1.0 and fit a circular model. We find that the best-fit circular model has a diameter of the geometric mean of the two axes, and thus the model provides accurate results about the surface area viewed. We next model two extreme cases of surface spots. First we create a model sphere having a single small area with twice the normal albedo and 0.44 times the diameter of the disk at the sub-observer point, which would cause a $20 \%$ flux variation. We then attempt to model this object as a uniform sphere. The derived size is again approximately proportional to the half-total light diameter, such that the modeled size is $\sim 10 \%$ smaller than the true size. If instead this bright spot is placed $60^{\circ}$ from the observer on the limb, the modeled size is only $\sim 2 \%$ larger than the true size. Larger numbers of albedo spots will tend to make the disk more uniform and will cause fewer problems. We thus estimate that this effect causes an uncertainty of $10 \%$ in the final size derivation.

A final source of error, and the only observational process that we could not adequately test with our analysis of the 47 Tuc observations, is any effect caused by the motion of Quaoar over the individual exposures. Errors in the motion vector with which we convolve our model could cause inaccuracies in our final measurements. These motion vectors are accurately defined by fitting to the full motion of Quaoar over the full orbit; nonetheless, we explore the effects of these errors. When we rerun the entire analysis but introduce $10 \%$ random $x$ and $y$ offsets to the modeled motion vector of each image, we find that the final angular size measured is unchanged, though the scatter is slightly larger. We thus conclude that this final potential systematic error has no discernible effect on the derived angular size.

\section{CONCLUSION}

Our final estimate of the diameter of Quaoar is $1260 \pm$ $190 \mathrm{~km}$, with the error bars dominated by the systematic error of the unknown limb-darkening profile. The red albedo, based on an opposition red magnitude of $18.5 \pm 0.1$ (with the uncertainty due to the unknown phase of the light curve at the time of observations) is $0.092_{-0.023}^{+0.036}$, while the blue albedo, based on a measured blue magnitude of $19.76 \pm 0.01$ and assuming an opposition brightening of $0.15 \mathrm{mag} \mathrm{deg}^{-1}$, is $0.101_{-0.024}^{+0.039}$.

The red albedo is consistent with the two values measured for other KBOs from radiometry. Assuming the albedos of all three objects are identical (which is not known to be the case but is a plausible hypothesis), we infer an average albedo of $0.088_{-0.012}^{+0.021}$. This albedo is significantly larger than the canonical $4 \%$ that is assumed in many studies. It is not known if most other KBOs have this albedo or if these largest KBOs, which are the only ones currently measured, are different. In addition, these three measured KBOs are all members of the high-inclination hot classical population of the Kuiper belt (Brown 2001; Morbidelli \& Brown 2004), whose members are distinguishable in color (Trujillo \& Brown 2002) and also maximum size (Levison \& Stern 2001; Morbidelli \& Brown 2004) from the low-inclination cold classical population, so their albedo properties might also be expected to be different. Assuming this albedo holds for large objects, the four intrinsically brightest KBOs-Quaoar, $2002 \mathrm{AW}_{197}$, Ixion, and Varuna-have diameters of 1290, 960, 960, and $800 \mathrm{~km}$, respectively. Quaoar would thus be the largest known minor planet and the largest object found in the solar system since the discovery of Pluto. While our current understanding of the albedos and sizes of KBOs is highly biased, continued application of this technique and the technique of millimeter radiometry, and the future use of SIRTF thermal measurements, will soon begin to provide the first hints of any interesting population properties of sizes and albedos of the objects in the Kuiper belt.

We would like to thank the director and staff at STScI for providing the opportunity to make these measurements and the assistance in making them happen. In particular, without prodding from Ian Griffin we would not have taken this second set of data, which allowed us to shrink the random-error bars by a factor of 6 . We would also like to thank John Krist for his excellent comments as a referee for this paper. This research has been supported by grant GO-9678 from STScI and by NASA Planetary Astronomy.

\section{REFERENCES}

Brown, M. E. 2001, AJ, 121, 2804

Jewitt, D., Aussel, H., \& Evans, A. 2001, Nature, 411, 446

Krist, J., \& Hook, R. 2001, The Tiny Tim User's Guide (ver. 6.0; Baltimore: STScI)

Levison, H. F., \& Stern, S. A. 2001, AJ, 121, 1730

Margot, J.-L., Trujillo, C., Brown, M. E., \& Bertoldi, F. 2002, BAAS, 34, 871
Morbidelli, A., \& Brown, M. E. 2004, in Comets II, ed. M. Festou, H. U. Keller, $\&$ H. A. Weaver (Tucson: Univ. Arizona Press), in press

Noll, K. S., Weaver, H. A., Storrs, A. D., \& Zellner, B. 1995, Icarus, 113, 353

Trujillo, C. A., \& Brown, M. E. 2002, ApJ, 566, L125 2004, in preparation 